\title{
Patio 2.12: Vivienda prefabricada, sostenible, autosuficiente y energéticamente eficiente. Participación en la competición Solar Decathlon Europe 2012
}

\author{
Patio 2.12: Prefabricated, sustainable, self-sufficient and energy efficient \\ house. Participation in the 2012 Solar Decathlon Competition \\ F. J. Terrados-Cepeda $^{(*)}$, L. Baco-Castro ${ }^{(*)}$, D. Moreno-Rangel ${ }^{(*)}$
}

\section{RESUMEN}

El prototipo Patio 2.12 fue la propuesta del equipo Andalucía Team para la competición Solar Decathlon Europe 2012 (SDE 2012), celebrada en Madrid en septiembre de 2012, en la que recibió hasta 9 premios, entre los que figura el de Eficiencia Energética.

\begin{abstract}
El equipo, integrado por profesores y alumnos de cuatro universidades andaluzas, trabajó durante dos años en el diseño y construcción de un prototipo de vivienda basada en la eficiencia energética, en la integración arquitectónica de los sistemas solares, en la prefabricación de escala intermedia y en una reinterpretación de la forma de vivir y construir de la tradición mediterránea. Patio 2.12 pretende ser un compendio de energía, sostenibilidad y mecanismos de acondicionamiento pasivo.
\end{abstract}

Palabras clave: Vivienda prefabricada; vivienda sostenible; competición; eficiencia energética; energía solar; acondicionamiento pasivo.

\section{ABSTRACT}

The prototype "Patio 2.12" was Andalucía Team's proposal for the 2012 Solar Decathlon Competition (SDE 2O12), that was held in Madrid during September 2012, where it was awarded several prizes like the first one in Energy Efficiency.

The team comprised a group of teachers and students from four Andalusian Universities. It worked for two years in the design and construction of a housing prototype that could be an example of several concepts at the same time: energy efficiency, architectural integration of solar systems, intermediate scale of prefabrication and a today's interpretation of traditional Mediterranean way of living and building. Patio 2.12 was intended to be a compendium of energy, sustainability and passive conditioning devices.

Keywords: Prefabricated housing; sustainable housing; competition; energy efficiency; solar energy; passive conditioning.

(*) Universidad de Sevilla (España).

Persona de contacto/Corresponding author: jterrados@us.es (F.J. Terrados-Cepeda)

Cómo citar este artículo/Citation: Terrados-Cepeda, F. J., Baco-Castro, L., Moreno-Rangel, D. (2015). Patio 2.12: Vivienda prefabricada, sostenible, autosuficiente y energéticamente eficiente. Participación en la competición Solar Decathlon Europe 2012. Informes de la Construcción, 67(538): eo88, doi: http://dx.doi.org/10.3989/ic.13.138.

Licencia / License: Salvo indicación contraria, todos los contenidos de la edición electrónica de Informes de la Construcción se distribuyen bajo una licencia de uso y distribución Creative Commons Reconocimiento no Comercial 3.o. España (cc-by-nc). 


\section{INTRODUCCIÓN}

La construcción industrializada tiene la necesidad de incorporar las cuestiones medioambientales y sociales que son características de nuestro tiempo, tales como el ahorro, la eficiencia, la mejora del confort, la personalización de los productos, etc.

Superada ya la etapa en la que lo principal era la producción masiva, la industrialización debe funcionar con nuevas dinámicas. Como señalan Ruiz Larrea et al. (1) en relación con el futuro de la construcción industrializada, «es necesario proponer soluciones transversales que impliquen a la Universidad, la profesión, la propia industria y la Administración». En esto debieron estar pensando los que, hace años, concibieron la competición de casas solares Solar Decathlon, cuyos participantes, las universidades, requieren del apoyo de la industria y de las administraciones para competir mediante prototipos de viviendas prefabricadas, eficientes y confortables.

\section{PRESTIGIOSA COMPETICIÓN INTERNACIONAI}

\subsection{Solar Decahtlon}

Es una prestigiosa competición internacional organizada por el Departamento de Energía de los Estados Unidos que premia la casa solar energéticamente más eficiente, sostenible y confortable. Se comenzó a celebrar en Washington en 2002 y constituye una excelente plataforma de difusión del conocimiento y aplicaciones de la eficiencia energética en el ámbito de la vivienda.

Los participantes españoles en Solar Decathlon 2005 ya explicaron en este medio (2) los principios básicos que sustentan el espíritu de la competición. Varios años más tarde, estos principios siguen siendo:

- Fomentar los sistemas de producción de energía a partir del sol para realizar, de manera confortable, todas las tareas que tienen lugar en la vivienda.

- Concienciar a la sociedad de los beneficios de las tecnologías solares, así como de sus posibilidades de integración arquitectónica.

- Estimular la investigación y desarrollo en energías renovables y eficiencia energética en edificación.

- La investigación en los sistemas de acondicionamiento pasivo y las cuestiones que afectan a la sostenibilidad y la huella ecológica, que, con el transcurso del tiempo, se ha incorporado como un objetivo cada vez más esencial en la competición.

Además, su repercusión en renovar los sistemas docentes en las escuelas de Arquitectura e Ingeniería, que se encuentran con una herramienta de gran potencial para generar formas de aprendizaje, colaboración interdisciplinar y entrenamiento en la resolución de problemas que serían inaccesibles por otros medios (3).

\subsection{Solar Decahtlon Europe 2012}

Fue ésta la séptima edición de Solar Decathlon y la segunda en su versión europea. La primera versión europea tuvo también lugar en Madrid, organizada en 2010 por el Ministerio de Vivienda del Gobierno de España junto con el Departa- mento de Energía de los Estados Unidos y la colaboración en la gestión del proceso completo a cargo de la Universidad Politécnica de Madrid (4). Desde el sur de España, la universidad de Sevilla tuvo ocasión de participar en aquella ocasión con el prototipo Solarkit (5).

En la competición de 2012 tuvieron representación América, Asia, África y Europa, participando cuatro equipos españoles: Universidad Politécnica de Cataluña, Universidad del País Vasco, Universidad Cardenal Herrera de Valencia y Andalucía Team, formado por cuatro universidades andaluzas (Escuelas Superiores de Arquitectura de Sevilla, Granada y Málaga y la Escuela Politécnica de Jaén).

El evento se desarrolló en el escenario Puerta del Ángel de la Casa de Campo de Madrid, donde dieciocho de los veinte equipos seleccionados consiguieron montar su prototipo de vivienda a tiempo para participar en el certamen (6).

En esta ocasión, las 10 pruebas recogidas en las normas de la competición fueron (7):
1. Arquitectura
2. Ingeniería y construcción
3. Eficiencia energética
4. Balance de energía eléctrica
5. Condiciones de confort
6. Funcionamiento de la casa
7. Comunicación y concienciación social
8. Industrialización
9. Innovación
10. Sostenibilidad

La asignación de la puntuación se basaba en varios criterios previamente establecidos: la monitorización de parámetros medibles (como el consumo eléctrico, la temperatura, la concentración de $\mathrm{CO}_{2}$ en el aire, etc.), el funcionamiento de los sistemas de la vivienda (los electrodomésticos o los sistemas de producción de agua caliente) y el fallo de jurados especializados y de carácter internacional.

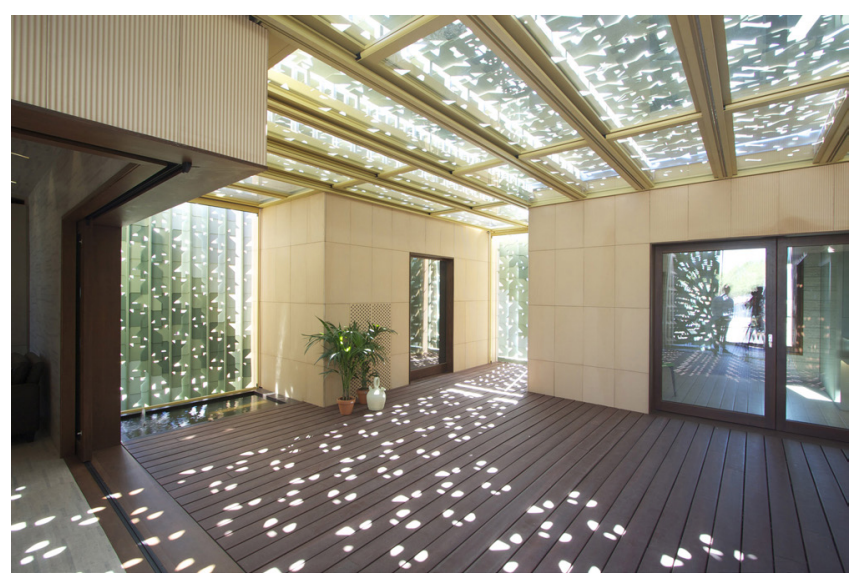

Figura 1. Vista interior de Patio 2.12 durante la celebración de SDE 2012. (Fotografía de SDE 2012).

\section{UN PROYECTO DE EQUIPO}

Andalucía Team es un equipo interuniversitario y multidisciplinar integrado por investigadores y alumnos de Arquitec- 
tura, Ingeniería y Comunicación. En el equipo quedaron integradas las aportaciones de cuatro universidades andaluzas.

- La Universidad de Sevilla aportó la experiencia de su participación en Solar Decathlon 2010, con el prototipo Solarkit, y los logros de algunos de sus investigadores en la vivienda prefabricada, (8).

- La Universidad de Jaén ofreció sus avances en la investigación sobre sistemas solares.

- La Universidad de Málaga contribuyó con su especialización en las tecnologías e instalaciones de la vivienda.

- La Universidad de Granada participó con su experiencia en la investigación en optimización estructural y constructiva.

El proceso de participación en SDE 2012 se extendió durante más de dos años, desde la solicitud de participación al concurso, en octubre de 2010, hasta la entrega de la última documentación requerida por la organización tras la competición, en diciembre de 2012, pasando por el montaje del prototipo en Madrid en septiembre de 2012 (Figura 1). La relación directa con los productores fue clave para hacer realidad el prototipo (9).

\section{PATIO 2.12: UNA VIVIENDA PREFABRICADA, MEDITERRÁNEA Y EFICIENTE}

Patio 2.12, con sus $70 \mathrm{~m}^{2}$ útiles (Figura 2), optó por proponer una revisión contemporánea de la arquitectura tradicional andaluza, a la que se incorporan las nuevas tecnologías relacionadas con la producción y el ahorro de energía. Capaz de producir más de tres veces la energía eléctrica consumida (430,87 kWh generada frente a 136,85 kWh consumida), el prototipo hizo de las estrategias pasivas para acondicionamiento climático uno de los principales argumentos de su diseño y construcción.

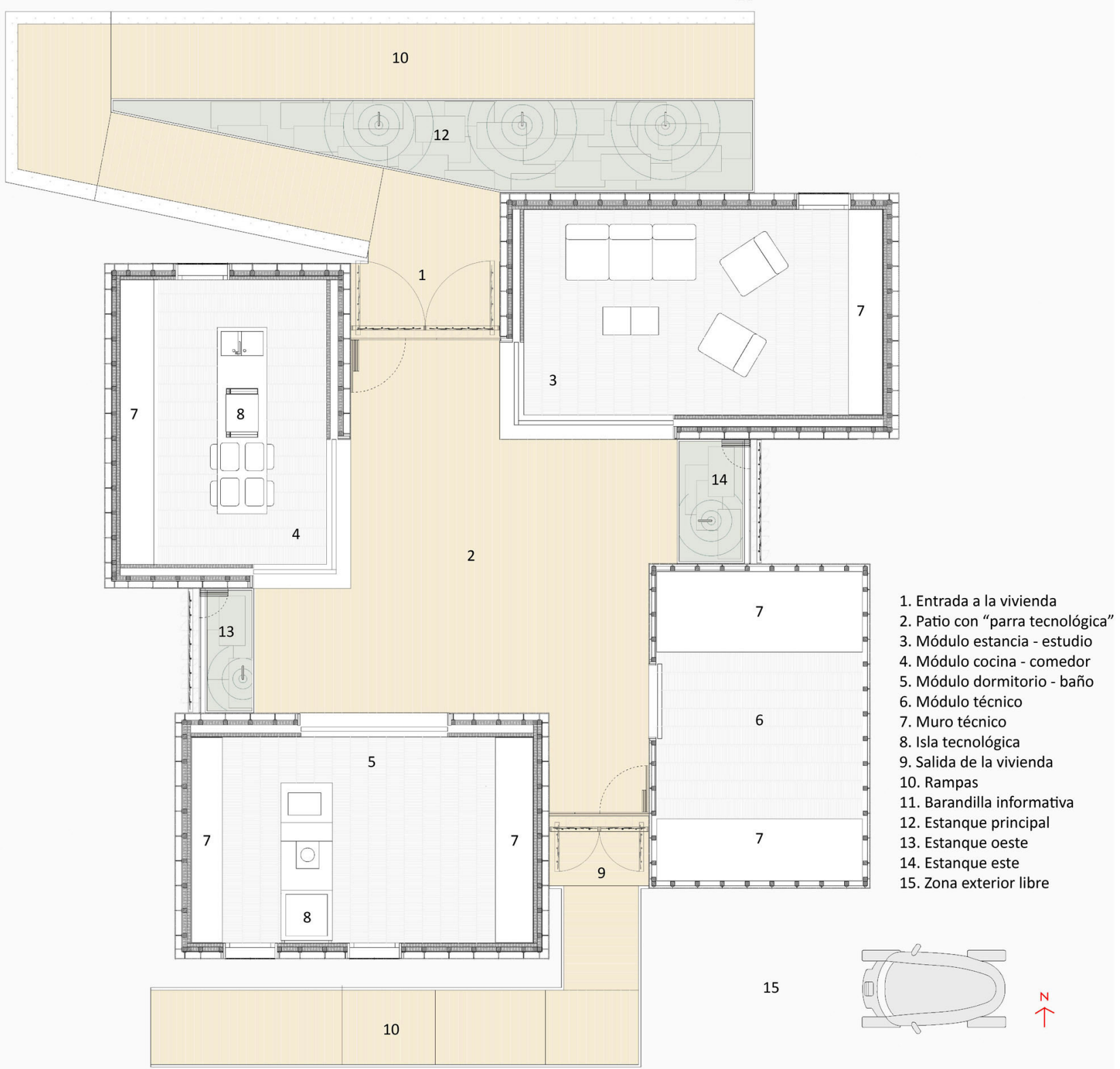

Figura 2. Planta de la vivienda Patio 2.12. 


\subsection{La arquitectura en Patio 2.12}

Los rasgos principales de la propuesta arquitectónica pueden sintetizarse como sigue:

- Patio 2.12 reinterpreta los espacios domésticos mediterráneos. El patio mediterráneo todavía puede considerarse un espacio arquitectónico ideal para relacionar la vivienda con el clima y el entorno mediterráneo (10). Las condiciones ambientales de los patios vernáculos: soleamiento, sombra, ventilación, humedad e incluso enfriamiento evaporativo (11), se recrean en nuestro prototipo con medios tecnológicamente avanzados, como es el caso de la llamada "parra tecnológica», que consiste en un sistema de doble piel, una de vidrio y otra en forma de lamas regulables, que consigue, a elección del usuario, condiciones adaptadas a cada estación del año: sombra ventilada, espacio solar, etc.

- Patio 2.12 es un kit de módulos prefabricados. El espacio doméstico propuesto no es la suma de habitaciones convencionales sino un conjunto de pabellones prefabricados mayores que una habitación, los cuales contienen cada uno de ellos una agrupación de usos compatibles (estancia-estudio, dormitorio-baño, cocina-comedor, etc.). El esquema de células mínimas habitables alrededor de un patio interior propio de algunas regiones mediterráneas (12) se reproduce aquí mediante la suma de pequeños lofts, cuyo número se puede incrementar cuando se crea conveniente, cambiar su posición o incluso transformar su uso en función de los requerimientos familiares. Este mismo esquema también ha sido interpretado con éxito por la arquitectura moderna en climas cálidos, el caso más claro son las propuestas de Charles Correa (13).

El tamaño de los pabellones prefabricados es el máximo posible para su transporte por carretera. Se relacionan entre sí a través del patio, un elemento flexible, que se construye in situ mediante elementos modulares semi-prefabricados. La prefabricación semi-cerrada -frente al otro extremo, la prefabricación abierta o «sutil», en la terminología de J. Salas (14) - es la única opción si se da prioridad a obtener un tiempo mínimo de montaje.

- Las «islas tecnológicas» y los «muros técnicos» cualifican el espacio interior de los módulos prefabricados. Los pabellones o módulos habitables son neutros, pudiendo acoger, en principio, cualquier función. Para adecuarse a un uso concreto, su interior se configura con la combinación de otros elementos prefabricados de menor escala, como si se tratase ahora de un kit de muebles (15). Éstos son de dos tipos: las llamadas «islas tecnológicas» y los llamados «muros técnicos».

Las «islas tecnológicas» son cápsulas exentas y compactas que contienen todos los elementos de instalaciones que dan servicio a la cocina o al cuarto de baño. En ambos casos, se ha experimentado con la búsqueda del máximo aprovechamiento del espacio en su diseño. Se conciben como contenedores de tecnología que incluyen novedosos inodoros, campanas extractoras escamoteables, etc. Los «muros técnicos» son piezas en forma de armario que se adosan a las paredes de cada módulo habitable, ocupándola completamente. Contienen dispositivos de almacenaje y un conjunto de instalaciones integradas, que permiten el buen funcionamiento del módulo (Figura 3).

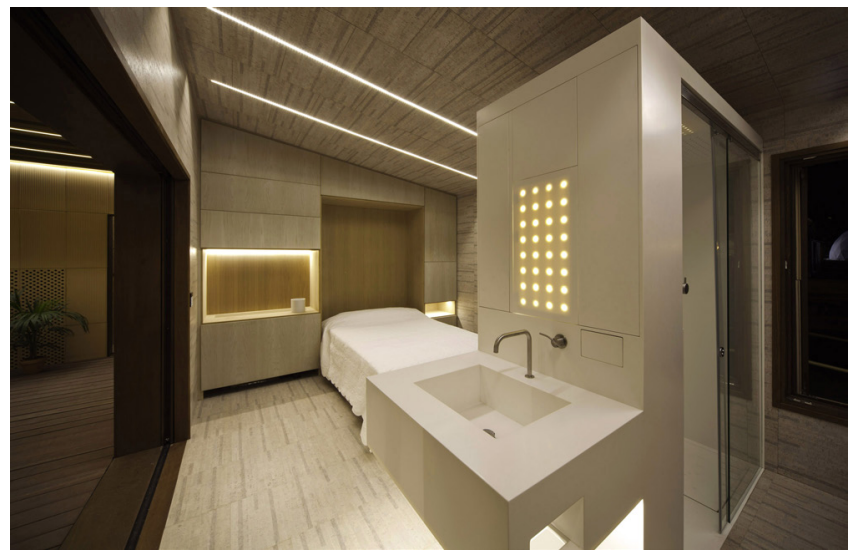

Figura 3. Vista interior del dormitorio - baño de Patio 2.12. (Fotografía de Pedro Ugarte).

- El prototipo no deja huella en el terreno. Cada uno de los módulos habitables prefabricados se deposita sobre el terreno por medio de seis soportes puntuales regulables, sin necesidad de cimentación.

- La integración arquitectónica de los sistemas activos es una de las principales características del prototipo. Todos los paneles solares están instalados con la inclinación de la cubierta del prototipo, quedando integrados en ésta como una capa exterior del cerramiento, sin comprometer el concepto de diseño de la vivienda (Figura 4).

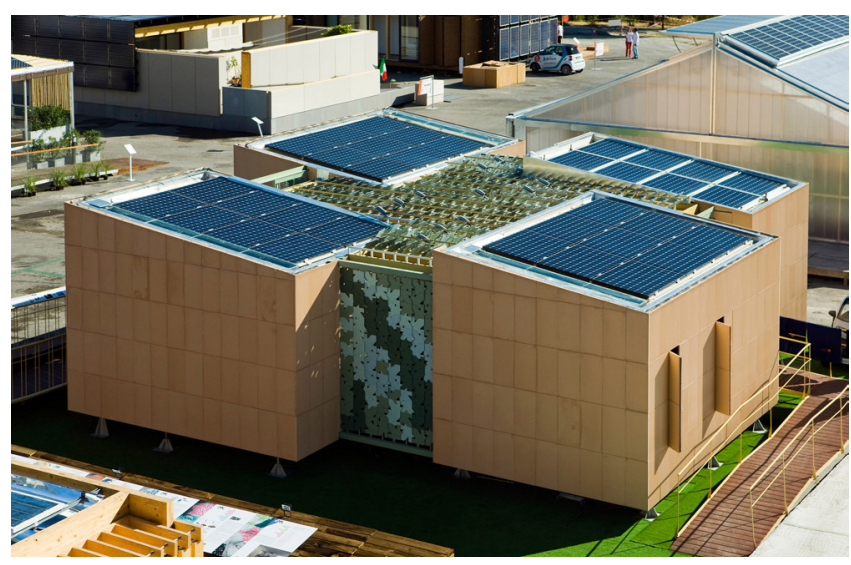

Figura 4. Vista del prototipo Patio 2.12 durante la celebración de SDE 2012. Madrid, septiembre de 2012. (Fotografía de SDE 2012).

\subsection{La construcción en Patio 2.12}

Los rasgos principales del sistema constructivo se pueden sintetizar como sigue:

- Toda la estructura de los módulos es de un mismo tipo: entramado difuso tipo balloon frame.

La estructura del prototipo es capaz de soportar las tensiones originadas en el transporte, el izado y el apoyo en plots sobre el terreno, de manera que no quede huella en el lugar donde se coloque. La estructura de los módulos habitacionales se configura con un forjado de perfiles metálicos huecos sobre el que se apoya un entramado de piezas de 
madera de pequeña sección, que resuelven la estructura vertical y de cubierta.

Los perfiles metálicos del forjado de suelo son: un anillo perimetral de tubo cuadrado de $200.200 .8 \mathrm{~mm}$ y viguetas en las dos direcciones de 200.80.3 mm.

La estructura vertical se genera a partir de pilares de madera maciza de $70 \times 70 \mathrm{~mm}$ colocados cada $40 \mathrm{~cm}$.

La estructura de cubierta se resuelve con viguetas de madera maciza $140 \times 70 \mathrm{~mm}$, colocadas cada $40 \mathrm{~cm}$ y viga perimetral de igual sección, o superior, en caso de solicitaciones estructurales mayores como la del vuelo en esquina en los módulos de salón, cocina y dormitorio.

- La envolvente de Patio 2.12 fue diseñada con un concepto de piel flexible que puede acumular o disipar el calor según convenga, cumpliendo el CTE-HE ahorro de energía (2006), así como el CTE-HS salubridad (2006) y CTE-HR Protección frente al ruido (2006). La transmitancia de los muros es bastante baja $\left(0,20 \mathrm{~W} / \mathrm{m}^{2} \mathrm{~K}\right)$, conseguida en paralelo a la optimización de su peso, con el objetivo de contar con un prototipo prefabricado fácilmente transportable.

\section{A. MÓDULOS HABITACIONALES \\ (Cocina, salón y dormitorio)}

\section{$\underline{\text { Envolvente vertical }}$}

La evolvente vertical de los módulos habitacionales de salón, cocina y dormitorio se compone de interior a exterior:

Acabado de paneles de corcho pegado. Wicanders. E: $3,5 \mathrm{~mm}$.

Barrera de Vapor. Tyvek VCL SD2, de DuPont.

Panel de thermochip ENERGY. E: $59 \mathrm{~mm}$. Conductividad: $0,029 \mathrm{~W} / \mathrm{mK}$.

Aislamiento lana mineral natural entre pilares. KNAUF

INSULATION Panel plus. TP 138. E: $80 \mathrm{~mm}$.

Conductividad: $0,032 \mathrm{~W} / \mathrm{mK}$.

Panel de OSB. E: $12 \mathrm{~mm}$.

Lámina impermeabilización Tyvek Fachada de DuPont.

Lámina de aislamiento reflexivo.

Cámara de aire ventilada. E: $100 \mathrm{~mm}$.

Pieza cerámica porosa (Acabado).

Sistema de riego de la pieza cerámica.

En la fachada norte, donde la incidencia solar es menor, se ha diseñado un sistema de riego de la cerámica con el objetivo de inducir la evaporación de la misma, para refrescar el aire que circula por la cámara. En la parte inferior del cerramiento, unas compuertas motorizadas controladas por domótica permiten la entrada a la estancia del aire de la cámara cuando su temperatura permita superar las cargas interiores consiguiendo la temperatura de confort ( $23-25^{\circ} \mathrm{C}$ según la competición).

\section{Envolvente horizontal. Cubierta}

La cubierta de los tres módulos antes mencionados, de interior a exterior, se compone de:

Acabado de paneles de corcho pegado. Wicanders. E: $3,5 \mathrm{~mm}$.
Falso techo de cartón yeso con perfilería oculta. KNAUF.

Aislamiento en cámara de falso techo. KNAUF INSULATION Panel plus. TP 138. E: $50 \mathrm{~mm}$.

Conductividad: $0,032 \mathrm{~W} / \mathrm{mK}$.

Panel de OSB. E: $12 \mathrm{~mm}$.

Aislamiento entre viguetas de madera. KNAUF INSULATION Panel plus. TP 138. E: $140 \mathrm{~mm}$.

Conductividad: $0,032 \mathrm{~W} / \mathrm{mK}$.

Panel sobre viguetas de madera de thermochip ENERGY. E: $79 \mathrm{~mm}$. Conductividad: $0,029 \mathrm{~W} / \mathrm{mK}$.

Impermeabilización de SIKA.

Cámara de aire de $9 \mathrm{~cm}$.

Módulos fotovoltaicos de Sun Power sobre estructura de perfiles de aluminio.

Puertas y ventanas de carpintería de madera y vidrio $8+12$ (con argón) $+4+12$ (con argón) +8 mm. Tipo Multipact 44.1 Neutralux S.

\section{Envolvente horizontal. Suelo}

El suelo de los módulos habitacionales, de interior a exterior, se compone de:

Suelo flotante de corcho. Flotating floor. Corkcomfort de Wicanders. E: $15 \mathrm{~mm}$.

Barrera de Vapor. Tyvek VCL SD2, de DuPont.

Panel sobre viguetas de madera de thermochip ENERGY. E: $59 \mathrm{~mm}$. Conductividad: $0,029 \mathrm{~W} / \mathrm{mK}$.

Aislamiento entre viguetas de metálicas. KNAUF INSULATION Panel plus. TP138. E: $200 \mathrm{~mm}$.

Conductividad: $0,032 \mathrm{~W} / \mathrm{mK}$.

Perfiles huecos de acero rellenos con Supafil, aislante de KNAUF INSULATION.

\section{B. PATIO}

$\underline{\text { Suelo }}$

El patio cuenta con un suelo técnico elevado con perfilería metálica y apoyos puntuales, dejando luces mínimas de $80 \mathrm{~cm}$ entre ellos.

Bajo el suelo técnico se colocan los depósitos de agua y el anillo de instalaciones, que conectan las instalaciones entre los cuatro módulo habitacionales.

Sobre el entramado elevado se coloca una solería tablas de composite de madera y plástico reciclado (WPC).

\section{Techo}

El techo del patio es modular y se apoya sobre los módulos habitacionales. Se compone de 11 cajones de $80 \mathrm{~cm}$ de ancho y longitud variable. Son elementos totalmente prefabricados en taller y se componen de:

Estructura de aluminio: Marco rectangular perimetral de aluminio que lleva incluidas las guías de corredera de las ventanas, el canalón y la caja con motores.

Ventana horizontal corredera motorizada, con perfilería de aluminio y vidrio Stadip 4+4 $\mathrm{mm}$ tipo Planitherm de Saint Gobain. 
Superiormente, la protección solar motorizada, con composite de aluminio pintada al silicato recortado con forma de hoja de parra y fijado a ejes metálicos de $80 \mathrm{~cm}$ de longitud.

\section{$\underline{\text { Laterales }}$}

Los laterales del patio se resuelven con:

Puertas de vidrio sin carpintería, tipo cortina de vidrio.

Protección solar con composite de aluminio similar al techo.

- Los materiales son sostenibles (42,35\% en peso del total) como la madera y la cerámica; reciclables $(46,70 \%)$ como el vidrio y el acero; y reciclados $(4,25 \%)$ como el corcho natural elegido para el acabado interior de la vivienda y las tablas de WPC empleadas en el patio.

Los elementos singulares de cocina y baño son piezas compactas prefabricadas insertadas en el interior de los módulos de Patio 2.12. Ambas se concibieron como contenedores tecnológicos que acogen equipos domésticos de última generación, realizados sobre una subestructura de perfiles de acero galvanizado revestido con un material sintético compuesto de resinas (Corian de Dupont), especialmente útil para los espacios húmedos.

Los llamados «muros técnicos», situados también en el interior de los módulos, pero esta vez adosados a los laterales con orientación este y oeste, están formados por tableros de DM acabados en madera natural de roble barnizada al agua. Tienen estantes y cajones para el almacenamiento, pero también contienen equipos específicos según el caso: fancoils para climatización, compuertas para la admisión de aire del sistema de ventilación natural, iluminación LED, equipos para el abastecimiento y depuración del agua, dispositivos para el control del sistema eléctrico, etc.

- El agua también es un material constructivo del prototipo. Participa de la configuración ambiental de la vivienda, así como de los sistemas de regulación térmica de la misma, tanto activos como pasivos. En el perímetro de la vivienda se disponen tres estanques, uno de ellos de mayor dimensión que los otros dos. Todos ellos tienen una fuente, cuyo rumor y frescura dan una sensación agradable al patio. El estanque principal también es usado para el sistema de climatización, en el circuito de pre-enfriamiento gratuito o pre free-cooling. Situado al norte y mayoritariamente en sombra, el agua de este estanque principal se puede utilizar, siempre que su temperatura sea inferior a $16{ }^{\circ} \mathrm{C}$, para realizar un enfriamiento gratuito de las estancias. Se genera un circuito de climatización primario entre el agua del estanque y un intercambiador de placa, de mínimo consumo eléctrico, situado bajo el patio. Desde ahí, parte un circuito secundario hasta cada uno de los fancoils, pasando por una batería adicional colocada en el conducto de aire de entrada al fancoil.

\subsection{Los sistemas de instalaciones en Patio 2.12}

En el prototipo Patio 2.12, todas las instalaciones se concentran en:

- El módulo técnico: las máquinas generales (bomba de calor, cuadros generales, etc.)
- El armario e islas de cada módulo habitacional: los elementos periféricos (fancoils, cuadros secundarios, etc.)

La conexión entre los módulos habitacionales se realiza mediante el anillo instalado debajo del suelo del patio, donde también se encuentran algunos depósitos de almacenamiento de agua.

Las instalaciones más destacadas del prototipo Patio 2.12 son:

- La instalación fotovoltaica de Patio 2.12 propone dos tipos de sistemas conectados a red con potencias de $2 \mathrm{kWp}$ y 2,5 kWp en función de la superficie de cubierta con la que cuenta cada módulo habitable. En total, son cuatro sistemas ( 2 de 2 kWp y 2 de $2,5 \mathrm{kWp}$ ), cada uno de los cuales puede funcionar independientemente del resto. La suma de la potencia de los inversores es de $9 \mathrm{kWp}$ y la potencia total del generador fotovoltaico es de $11,3 \mathrm{kWp}$ en condiciones estándares. La conexión con el sistema general de la casa y con la red exterior está situada en el llamado «módulo técnico». Los principales componentes de esta instalación son: módulos fotovoltaicos de silicio cristalino con tecnología de células de contacto posterior (consiguen una eficiencia de conversión superior al $20 \%$ ), inversores de conexión a red de última generación y de alto rango de conversión (aseguran la independencia de la red eléctrica gracias a técnicas de alta frecuencia) y sistema «Sunny-Back-UP» para almacenamiento y gestión de la energía.

- La instalación solar térmica para la producción de agua caliente sanitaria cubre más del $70 \%$ de la demanda anual, conforme al CTE-HE-4 (2006). Tras investigar las diversas soluciones posibles, finalmente se optó por un sistema híbrido de paneles fotovoltaicos y colectores solares térmicos. La instalación se compone de 8 módulos híbridos colocados se sobre la cubierta inclinada del módulo técnico, con la misma apariencia que los módulos fotovoltaicos de las otras tres cubiertas. Este sistema permite utilizar la energía solar captada por las células fotovoltaicas además de calentar el agua que circula bajo éstas, por un serpentín perfectamente aislado y protegido. Se dispone de un depósito acumulador vertical situado en el módulo técnico de 300 litros, diseñado para mantener el agua a $60{ }^{\circ} \mathrm{C}$ y conectado al depósito que se calentará, en caso de necesidad, mediante la bomba de calor del sistema de climatización.

- El sistema domótico permite mejorar la eficiencia energética y la calidad de vida de los usuarios. Es posible conocer, a tiempo real y mediante la colocación de un gran número de sensores y de bus KNX, las condiciones ambientales interiores y exteriores, de iluminación, de producción de energía, etc. Además, el sistema posibilita gobernar la climatización (activando o desactivando los distintos dispositivos o equipos), controlar la calidad del aire (mediante la apertura o cierre de compuertas), controlar la iluminación exterior e interior, monitorizar el consumo y la producción de energía y administrar todas las aplicaciones de la vivienda mediante terminales telefónicos y tabletas.

En el módulo técnico se encuentra el cuadro general de domótica, desde donde se controlan y gestionan todos los 
sistemas del prototipo. La instalación de domótica es paralela a la de electricidad, existiendo una pantalla de control integrada en el armario de cada módulo habitacional.

- Los sistemas de acondicionamiento térmico se ponen en marcha de manera gradual según las necesidades. Son de tres tipos, sistemas pasivos (que no consumen energía eléctrica), sistemas semi-activos (donde la energía eléctrica consumida es puntual y de rango inferior a 100W) y sistemas activos (que necesitan energía eléctrica durante su funcionamiento con alto grado de consumo).

\section{Sistemas Activos}

a. En el módulo técnico se encuentra la bomba de calor (marca Ciatesa), de condensación por aire.

Desde ella, parten las tuberías hasta los fancoils (Aqualis Inverter). El salón, la cocina y el dormitorio cuentan con un fancoil integrado en su armario. El sistema de pre freecooling diseñado hace pasar el agua del estanque principal por una batería de condensación previa a los fancoils.

Este sistema de climatización es el que garantiza alcanzar el rango de temperatura de 23 a $25^{\circ}$ con los que se puntúa el máximo en la prueba de Condiciones de Confort.

\section{Sistemas Semi-pasivos}

a. Se realiza un enfriamiento evaporativo del patio mediante una máquina evaporativa situada en el módulo técnico (marca Breezair) que impulsa aire humectado al patio. Este sistema se incluyó para utilizarlo durante las visitas del público.

b. Evaporación de la fachada cerámica. Desde el depósito bajo el patio, se bombea agua a la parte alta de los módulos habitacionales para regar, con sistema por goteo, las piezas cerámicas de las fachadas orientadas al norte de los módulos de salón y cocina.

Esta agua se evapora sólo hacia el exterior de la cerámica, refrescando la cámara de aire de fachada. Las rejillas motorizadas introducen el aire fresco al interior de la estancia, mejorando las condiciones de confort interior. Es un sistema de acondicionamiento semi-pasivo, que sólo se utiliza cuando las condiciones exteriores no son tan extremas y es posible llegar a la temperatura de confort.

c. Ventilación y sombreamiento del patio. El sistema domótico del prototipo activa los motores de las ventanas de vidrio y de las hojas de composite del techo del patio, para permitir ventilaciones y sombras en el patio, según unos sensores de temperatura exterior y la posición solar de cada momento.

\section{Sistema pasivo.}

Lo constituye la ventilación natural de las estancias, en cuyo interior, el aire caliente tiende a subir y salir por las rejillas situadas en la parte alta de los armarios. Estas rejillas están conectadas con una chimenea solar situada en cubierta. En la chimenea solar (un cajón cerrado superiormente por vidrio y con una piedra basáltica en su fondo) se producen convecciones que generan una succión del aire interior de las estancias.
El sistema domótico da orden de activación, de manera progresiva a los sistemas antes descritos:

- El patio permanece sombreado y ventilado en verano; y soleado y cerrado en invierno.

- El sistema evaporativo de la fachada norte, se activa cuando la temperatura interior está entre $23,5{ }^{\circ} \mathrm{C}$ y $24,8^{\circ} \mathrm{C}$.

- Las rejillas de ventilación conectadas a la chimenea solar se activan cuando el nivel de $\mathrm{Co}_{2}$ interior alcanza $1000 \mathrm{ppm}$.

- La máquina evaporativa del patio se activa cuando algún punto de la casa supera los $23,5{ }^{\circ} \mathrm{C}$ de temperatura y se desactiva alcanzados $\operatorname{los} 24,8^{\circ} \mathrm{C}$.

- El rango de operación del sistema hidrónico con fancoils es entre $24{ }^{\circ} \mathrm{C}$ y $24,8{ }^{\circ} \mathrm{C}$ en el modo de refrigeración, y entre $23,2{ }^{\circ} \mathrm{C}$ y $23,8^{\circ} \mathrm{C}$ para la calefacción.

\section{Reciclado de aguas grises}

Las aguas grises son almacenadas bajo el suelo del patio y de ahí bombeadas a la depuradora colocada en el módulo técnico. Una vez tratadas, las aguas son almacenadas también bajo el suelo del patio, para usarse luego en el riego.

La depuradora, diseñada específicamente para incluirse en el ámbito doméstico, recicla las agua grises reproduciendo los procesos de eliminación de contaminantes que se producen en los humedales naturales de flujo vertical. El agua circula para ser tratada de arriba abajo a través de varios sustratos de filtro de material inerte (de grava y arena, en cuya superficie se forma una película de bacterias para la oxidación biológica de los contaminantes) y de filtros de aireación (para mantener las condiciones aeróbicas en la biomasa). Finalmente, una luz ultravioleta desinfecta el agua.

En todo momento fue prioritaria la integración de todos los sistemas de instalaciones en un concepto arquitectónico unitario.

\subsection{La eficiencia energética en Patio 2.12}

La elección del patio central como tipología de vivienda, además de su eficiencia energética, tiene también un sentido cultural (16), de forma que se evoca el modo de vida tradicional mediterráneo. El «estilo de vida mediterráneo» fue también la seña de identidad del equipo Andalucía Team. Se optó por combinar mecanismos avanzados de eficiencia energética con estrategias de «sentido común». La eficiencia energética del prototipo se evidencia en:

- En la arquitectura del prototipo, ya que su organización de módulos habitables en torno a un patio permite:

Que el patio funcione como un espacio de amortiguación, ya que cada módulo habitacional se relaciona con el exterior a través del patio, actuando éste como un regulador térmico de la vivienda. El patio se convierte en un espacio habitable más de la vivienda, como en la tradición mediterránea, usando la fuerte ventilación y el control de la sombra para la temporada de calor, y actuando como colector solar al favorecer el efecto invernadero en la época de bajas temperaturas.

Las variaciones del factor de forma, ya que en el prototipo no existen límites fijos para el patio. El vidrio del cerramiento vertical y de la cubierta del patio puede abrirse o cerrarse, dependiendo de las condiciones meteorológicas 
existentes en cada momento. Esto supone que el FF (ratio entre la superficie de fachada y el volumen) varía en un rango de 1,4 en verano a 1,0 en invierno, de forma que el usuario será el que regule la vivienda para conseguir el óptimo en cada época del año.

- En la envolvente del prototipo, por su aislamiento térmico, su inercia térmica, su color y por la baja emisividad de los vidrios empleados. La envolvente se diseñó con una transmitancia térmica baja $\left(0,12-0,20 \mathrm{~W} / \mathrm{m}^{2} \mathrm{~K}\right.$, según hablemos de la cubierta o del cerramiento exterior) de acuerdo con los parámetros estándares de acondicionamiento pasivo de la vivienda, incorporando aislamiento de lana mineral de alta densidad $\left(100 \mathrm{~kg} / \mathrm{m}^{3}\right)$ entre los postes de la estructura tipo ballon frame de los módulos y colocando una lámina de aislamiento reflexivo previa a la cámara ventilada del cerramiento. La última capa del cerramiento de los módulos de Patio 2.12 está formada por piezas de cerámica. Su color es claro, que minimiza la absorción solar, al tiempo que la cámara ventilada reduce las sobrecargas por radiación solar directa. Las carpinterías de vidrio incluyen sistemas de rotura de puente térmico y un triple vidrio de baja emisividad $(8+12+4+12+8)$, con una transmitancia de $0,7 \mathrm{~W} / \mathrm{m}^{2} \mathrm{~K}$.

- En el uso medioambiental y arq uitectónico del agua, ya que en la tradición mediterránea, el agua va intrínseca en la idea del patio, no sólo ayuda a regular térmicamente el ambiente en dicha estancia, sino que además ofrece un cierto confort psicológico gracias al sonido que produce. El agua también está presente en el sistema evaporativo del patio, que consigue reducir la temperatura y recircular aire fresco con muy poco consumo de energía, permitiendo climatizar las estancias cuando las condiciones ambientales no sean extremas.

- En los sistemas pasivos del prototipo, que son el «efecto botijo» y la ventilación pasiva:

El «efecto botijo» consiste en el pre-enfriamiento de aire por evaporación para el caso de verano. La característica más reconocible de la envolvente del prototipo es su capa exterior compuesta por placas de cerámica porosas que le otorga el aspecto de un botijo. Y no es sólo por su aspecto sino por su funcionamiento. Las piezas cerámicas incluyen un sistema de riego capilar que enfría el aire en el interior de la cámara del cerramiento a través de un proceso de evaporación del agua. La cara interior de las placas se ha impermeabilizado por lo que se evita que la humedad traspase a la cámara para dar cumplimiento al CTE HS3 (2006). Este efecto produce un descenso de entre 5 y 10 grados de temperatura en la cámara en comparación con el exterior.

El aire fresco entra al interior de la estancia a través de unas compuertas motorizadas de aluminio de $15 \mathrm{~cm}$ de altura, situadas en la parte inferior del cerramiento y provistas de poliestireno extruido adherido en sus lamas, evitando así el puente térmico (Figura 5).

Para la ventilación pasiva, los módulos habitacionales cuentan también con otras compuertas conectadas con chimeneas solares localizadas en la cubierta, antes descritas.

La cámara de aire incluida en la fachada norte, la chimenea solar, el efecto del sistema evaporativo del patio, así como la placa híbrida y la cámara de aire bajo las placas foto-

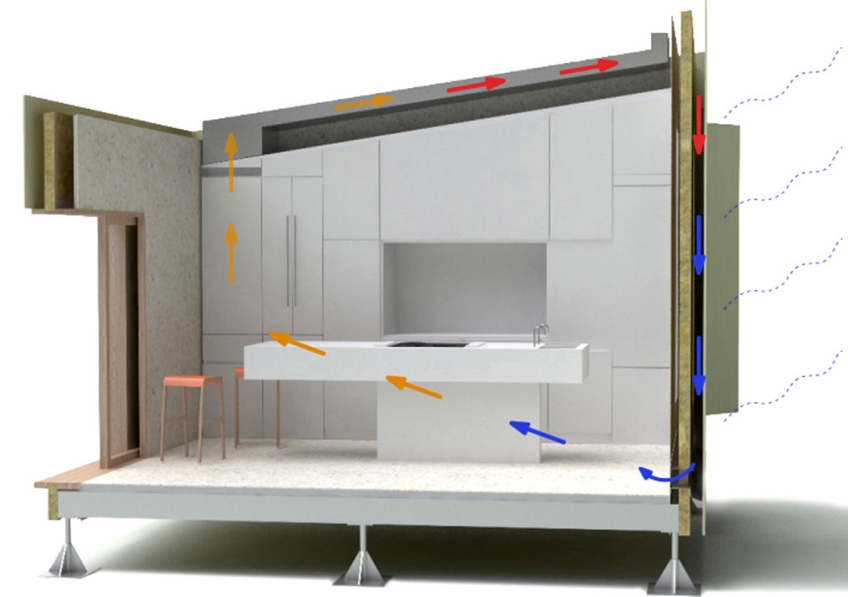

Figura 5. Esquema del funcionamiento del «efecto botijo» y la ventilación pasiva en el módulo cocina de Patio 2.12.

voltaicas fueron simuladas mediante programa de cálculo de elementos finitos (ANSYS) para dimensionarlos y comprobar su eficiencia respecto otros modelos que carecen de ellos. Mediante la simulación con el programa DesignBuilder se estudió el comportamiento pasivo en fase de diseño del edificio (Figura 6).

- En el balance eléctrico del prototipo, que es muy positivo gracias al rendimiento de la instalación fotovoltaica y al control del consumo en el prototipo (bastante bajo al emplear electrodomésticos de gran eficiencia, iluminación LED y un gran número de estrategias bioclimáticas que reducen significativamente el uso de energía eléctrica para el uso diario y confortable de la casa). Así, el balance eléctrico estimado del prototipo fue de $13.405,39 \mathrm{kWh} / \mathrm{a}$, con un consumo previsto de $127,88 \mathrm{kWh}$, y una producción de 320,27 kWh. Durante la competición, esta previsión fue mejorada y el balance eléctrico real del prototipo fue:

\section{Energía generada: 430,87 kWh}

Energía consumida: $136,85 \mathrm{kWh}$

Balance eléctrico con la red: $287,36 \mathrm{kWh}$ (inyectados a red).

- En el balance del ciclo de vida del prototipo, ya que tanto para la construcción como para la vida del edificio está presente el concepto del reciclaje. Muchos materiales de construcción proceden de procesos de reciclado industriales, o bien, se les dio un nuevo uso en el prototipo. En el caso del agua, se diseñó un sistema de reciclado del agua empleada en el funcionamiento normal de la vivienda. Además, el terreno donde se instale la casa quedará sin huella una vez retirado el prototipo, gracias su sistema de apoyo. En términos objetivos, el balance energético del ciclo de vida (calculado para 31 años) es de $+382.176,68 \mathrm{MJ}$.

\section{RESULTADOS DE LA COMPETICIÓN}

La participación de Andalucía Team en Solar Decathlon Europe 2012 superó las expectativas. Siendo uno de los equipos con un presupuesto más bajo, el prototipo fue el primero en construirse (hecho que reconoció la organización de la competición) y consiguió situarse entre los más puntuados desde 




el principio, quedando finalmente en segunda posición de la clasificación general, a tan solo 11 puntos de los 908,72 puntos del equipo ganador (Team Rhone Alpes). Asimismo, fue el equipo que más premios consiguió en las pruebas parciales, obteniendo incluso el premio del público visitante. La relación de premios obtenidos es la siguiente:

- 1er Premio al Favorito del Público

- 1er Premio en Comunicación y Conciencia Social

- 1er Premio en Eficiencia Energética

- 1er Premio en Balance de Energía Eléctrica

$-2^{\mathrm{O}}$ Premio en Sostenibilidad

$-2^{\circ}$ Premio del Green Building Council

$-2^{\circ}$ Premio en Innovación

- $3^{\text {er }}$ Premio en Ingeniería y Construcción

- $2^{\mathrm{O}}$ Premio Final Solar Decathlon Europe 2012

En cuanto a la monitorización del prototipo, cabe reseñar las temperaturas registradas (Figura 7) y el comportamiento eléctrico real de la casa durante la competición:

Las normas de la competición establecían que la temperatura interior en las estancias monitorizadas debía estar en el rango de 20 a $28^{\circ} \mathrm{C}$, obteniendo la mayor puntuación en el rango de 23 a $25{ }^{\circ} \mathrm{C}$. Si observamos las gráficas de la temperatura monitorizada en el salón y dormitorio, apreciamos que apenas se tuvieron registros fuera del rango puntuable. Durante los primeros días de la competición se mantuvo en el rango de 22 a $26{ }^{\circ} \mathrm{C}$, bajando al rango de los 20 a $24{ }^{\circ} \mathrm{C}$ durante los últimos días, cuando la temperatura exterior sufrió un importante descenso. Esto, que repercutió en una menor puntuación durante estos días, evidenció el mejor comportamiento de la casa frente a las altas temperaturas y pudo haberse evitado si se hubieran instalado, tal como se había diseñado, los amor-
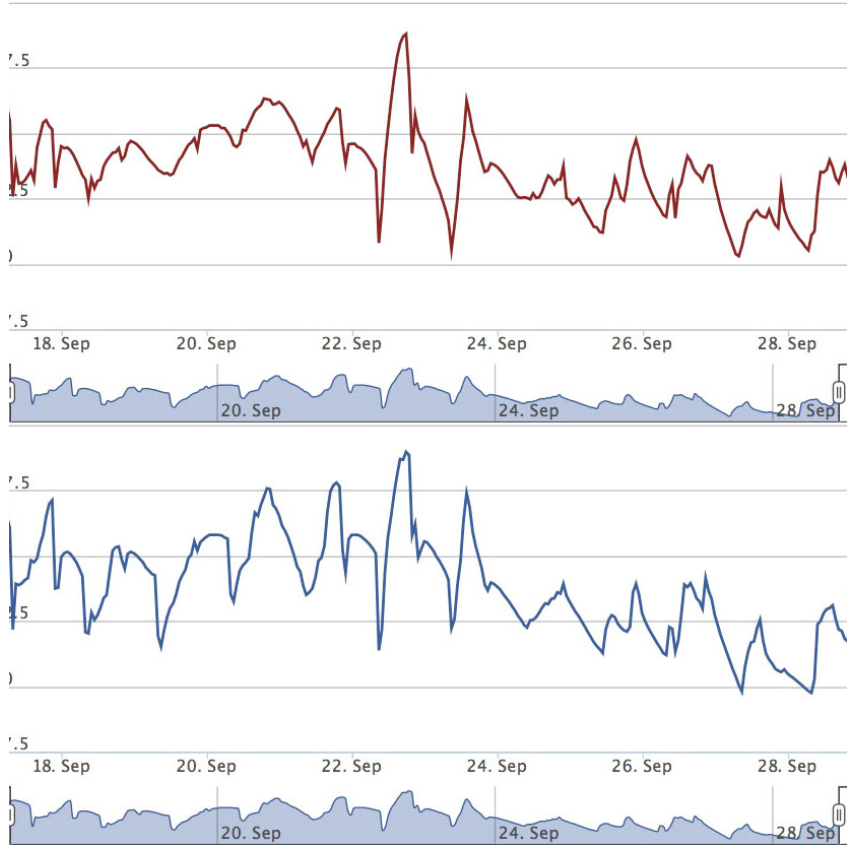

Figura 7. Temperatura interior en el salón (arriba) y el dormitorio (abajo) de Patio 2.12 durante la competición SDE 2012. (Gráfica de SDE 2012).

tiguadores regulables bajo el perímetro de las estancias, cerrando la cámara de aire bajo el suelo de éstas.

Por último, si comparamos el patrón de ambas gráficas, notamos que en el salón, para los días de calor, las fluctuaciones de temperatura entre el día y la noche fueron menores. 


\section{CONCLUSIONES}

El diseño y la construcción del prototipo Patio 2.12 tuvo como uno de sus objetivos principales la consecución de un sistema arquitectónico lo más eficiente posible que aunara para ello conceptos procedentes de la tradición residencial mediterránea y avances tecnológicos en el campo de la prefabricación, las instalaciones y la gestión de la energía.

El grado de consecución de estos objetivos puede calibrarse en la transcripción del informe del jurado que otorgó al equipo Andalucía Team el primer premio en Eficiencia Energética, uno de los más codiciados de la competición (7):

«El primer premio es un prototipo con un concepto innovador de la construcción de viviendas con módulos habitables prefabricados, que tiene un claro potencial de mercado.

Extremadamente bien armonizados los elementos constructivos y los espacios de las casas tradicionales, sofisticadamente enriquecidos con la comprensión crucial de los vínculos inextricables entre las características del cerramiento y la construcción de las relaciones entre el interior y el exterior que se resuelven en este edificio a través de su tradicional construcción en patio con su potencial comportamiento de alta eficiencia.

Además del innovador sistema de refrigeración evaporativa de la fachada, el edificio tiene un sistema fotovoltáico que tiene una doble función, cubierta y generación eléctrica. Los paneles solares situados en la cubierta de los módulos habitables crean una cámara de aire sobre pequeños soportes, con la inclinación adecuada para alcanzar la más alta eficiencia.

Crucialmente diseñado como un sistema eficiente activo/ pasivo, este edificio tiene potencial para desarrollar su vida como un saludable edificio de alta calidad en todos los aspectos del entorno interior».

En cuanto a la eficacia del sistema prefabricado previsto, que buscaba principalmente la mayor rapidez de montaje in situ (esencial para disponer de mayor tiempo de pruebas en la competición), da fe el haber conseguido la distinción que la competición otorgaba al equipo que finalizaba antes la casa, distinción que llevaba aparejada ser la vivienda que acogía a las personalidades el día de la inauguración.

El primer premio como Favorito del Público manifestó el éxito de la arquitectura del prototipo. Para los miles de visitantes anónimos, Patio 2.12 fue la mejor casa, su condición de prefabricada no impidió que se identificaran en sus espacios y los reconocieran como habitables y la situaran como la más apetecible para habitar.

El hecho de disponer, durante el período de monitorización, de una semana cálida, veraniega, y otra semana claramente más fría, otoñal, permitió calibrar el comportamiento de los sistemas pasivos, semi-pasivos y activos para diferentes condiciones exteriores. Los resultados mostraron un muy buen comportamiento en la semana cálida, donde no hubo necesidad de poner en funcionamiento los sistemas activos y donde el novedoso sistema evaporativo de fachada contribuyó a una refrigeración de casi nulo consumo energético. En la semana fría fue necesaria la puesta en funcionamiento de los sistemas activos. El comportamiento del patio como colector solar se vio penalizado por la inexistencia de cierre en la cámara de aire inferior de este patio (no se pudo construir el cierre neumático regulable previsto en el perímetro de esta cámara), lo que provocó ciertas fugas térmicas por el suelo elevado de tablas con juntas abiertas.

A la vista de los resultados y del desarrollo del proceso, la participación de nuestro equipo en SDE 2012 es un ejemplo de buena colaboración entre profesionales, universidades, industria y administraciones. En algunos casos, la industria se adaptó a los diseños iniciales y en otros, fueron retocados los diseños para ajustarlos a las posibilidades industriales. Ello fue posible gracias al empeño de investigadores, estudiantes, profesionales e industriales del mundo de la construcción, a los que desde este texto se quiere expresar el más sincero agradecimiento.

\section{REFERENCIAS}

(1) Ruiz-Larrea, C., Prieto, E., Gómez, A. (2008). Arquitectura, Industria y Sostenibilidad. Informes de la Construcción, 6o(512): 35-45, doi: http://dx.doi.org/ 10.3989/ic.08.037.

(2) Camaño-Martín, E., Neila-González, J., Jiménez-Leube, F.J., Egido-Aguilera, M.A., Uzquiano, M. J., Gómez-Osuna, J.M., Bedoya-Frutos, C., Magdalena-Layos, L., García-Santos, A. (2004). Viviendas solares autosuficientes: participación de la Universidad Politécnica de Madrid en el Concurso Solar Decathlon. Informes de la Construcción, 56(494): 35-46, doi: http://dx.doi.org/ 10.3989/ic.2004.v56.i494.447.

(3) Torres-Antonini, M. (2013). Building the future: The solar decathlon as education for future sustainbility leadership, Sustainability, 6(1): 48-56, doi: http://dx.doi.org/10.1089/sus.2013.9891.

(4) Vega-Sánchez, S. (2011). Solar Decathlon Europe 2010. Towards Energy Efficient Buildings. Madrid: 10 Action Project. Universidad Politécnica de Madrid

(5) Terrados-Cepeda, J. (2011). Solarkit: Una vivienda desmontable y energéticamente autosuficiente. Sevilla: Universidad de Sevilla.

(6) Vega-Sánchez, S. (2013). Solar Decathlon Europe. Improving Energy Efficient Buildings. Madrid: 10 Action Project. Universidad Politécnica de Madrid.

(7) Sd Europe-Solar Decathlon: http://www.sdeurope.org/

(8) Terrados-Cepeda, J. (2012). Prefabricación ligera de viviendas. Nuevas premisas. Sevilla: Instituto Universitario de Arquitectura y Ciencias de la Construcción - Universidad de Sevilla.

(9) Salas, I., Oteiza I., (2009). Estrategias divergentes de industrialización abierta para una edificación pretenciosamente sostenible. Informes de la Construcción, 61(513): 11-31, doi: http://dx.doi.org/10.3989/ic.08.050. 
(10) Vázquez-Gutiérrez, J. (1997). Arquitectura y Clima en Andalucía. Manual de diseño. Sevilla: Consejería de Obras Públicas y Transportes - Junta de Andalucía.

(11) Fathy, H. (1986). Natural energy and vernacular architecture. Principles and examples with reference to hot arid climates. EE.UU: University of Chicago.

(12) De Llorens-Durán, J.I., Soldevilla-Barbosa, A. (1994). Alternativas tipológicas a la vivienda convencional. Informes de la Construcción, 46(434): 5-25, doi: http://dx.doi.org/10.3989/ic.1994.v46.i434.1105.

(13) Correa, C. (2000). Housing and urbanisation. Londres: Thames \& Hudson.

(14) Salas, J. (2008). De los sistemas de prefabricación cerrada a la industrialización sutil de la edificación: algunas claves del cambio tecnológico. Informes de la Construcción, 6o(512): 19-34, doi: http://dx.doi.org/10.3989/ic.07.001.

(15) Terrados-Cepeda, J. (2012). El proyecto Arkit. La vivienda como kit de muebles. Proyecto, progreso, arquitectura, (6): 114-131.

(16) Blaser, W. (1997). Patios. 5000 años de evolución desde la antigüedad hasta nuestros días. Barcelona: Gustavo Gili. 